\title{
Coulomb blockade oscillations of conductance in the regime of strong tunneling
}

\author{
A. Furusakit and K. A. Matveev \\ Department of Physics, Massachusetts Institute of Technology, Cambridge, MA 02139
}

(April 4, 2021)

\begin{abstract}
We study the transport through a quantum dot coupled to two leads by singlemode point contacts. The linear conductance is calculated analytically as a function of a gate voltage and temperature $T$ in the case when transmission coefficients of the contacts are close to unity. As a function of the gate voltage, the conductance shows Coulomb blockade oscillations. At low temperatures, the off-resonance conductance vanishes as $T^{2}$, in agreement with the theory of inelastic co-tunneling. Near a resonance, the low-energy physics is governed by a multi-channel Kondo fixed point.
\end{abstract}

PACS numbers: 73.20.Dx, 73.40.Gk 
The Coulomb blockade of tunneling has recently become a subject of intensive studies 11. It is usually observed by measuring the conductance of a system of two bulk electrodes connected by tunnel junctions to a small conducting island. Tunneling of an electron into the island is accompanied by the increase of the energy of the system by $E_{C}=e^{2} / 2 C$, where $C$ is the capacitance of the island. At low temperatures $T \ll E_{C}$ this leads to the suppression of tunneling. This phenomenon is due to the discreteness of charge in the island, and can be suppressed by tuning a gate voltage to the point where the energies of the states with $n$ and $n+1$ electrons in the island are equal. At these points the energy gap related to the charging energy vanishes, and one observes peaks in conductance as a function of the gate voltage.

A popular realization [2] of the conducting island is a quantum dot, Fig. 1, created artificially in a two-dimensional electron gas (2DEG), and connected to the large areas of 2DEG (the leads) by quantum point contacts (QPC). In such a system, the transmission coefficient $\mathcal{T}$ of a QPC connecting the dot and an external lead can be controlled by changing a gate voltage. This opens a possibility for studying the Coulomb blockade effect in the strong-tunneling regime of $\mathcal{T} \rightarrow 1$, where one naively expects that the Coulomb blockade may not be observed because the number of electrons in a quantum dot is no longer well defined. Recent experiments indicate, however, that even when $\mathcal{T}$ is close to 1 the conductance shows periodic oscillations although the peaks are not well separated [3, 国. Most of theoretical work has been concentrated on the case of weak tunneling $(\mathcal{T} \ll 1)$, and only the equilibrium thermodynamic quantities have been discussed in the strong-tunneling limit [5]. The aim of this paper is to develop a theory of the transport through a quantum dot in the strongtunneling regime. We show that in the low-energy limit the conductance is renormalized to zero off resonance, and to $\sim e^{2} / h$ on resonance, yielding clear Coulomb blockade peaks even for the strong-tunneling case. We also derive analytic expressions for the conductance in some interesting limiting cases.

In the weak-tunneling limit there are two different mechanisms of low-temperature conductance. One contribution is due to real transitions of electrons between the leads and the dot [6]. If the gate voltage $V_{g}$ is not equal exactly to the nearest resonant value $V_{g}^{(n)}$, an energy cost $\Delta E \propto V_{g}-V_{g}^{(n)}$ is associated with such a transition. Since only an exponentially small fraction of electrons have energy $\Delta E$ at low temperatures, the conductance decays exponentially away from the peak:

$$
G=\frac{1}{2} \frac{G_{L} G_{R}}{G_{L}+G_{R}} \frac{\Delta E / T}{\sinh (\Delta E / T)} .
$$

Here $G_{L}$ and $G_{R}$ are the conductances of the left and right QPCs. At very low temperatures another mechanism of transport through the dot dominates []]. This mechanism, commonly referred to as the inelastic co-tunneling, corresponds to the second-order tunneling processes. At the first step an electron tunnels from the left lead to a virtual state in the dot, and the system acquires charging energy $\sim \Delta E$. At the second step, another electron tunnels from the dot to the right lead, thus finishing the process of the charge transfer and restoring the initial charge of the dot. In any such process, the original electron decays into three quasiparticles (an electron in the right lead and an electron-hole pair in the dot). Similarly to the problem of decay of a quasiparticle in Fermi liquid, this means that the tunneling rate is proportional to $T^{2}$ in the low-temperature limit, 


$$
G=\frac{G_{L} G_{R}}{6 G_{0}}\left(\frac{T}{\Delta E}\right)^{2}, \quad T \ll \Delta E,
$$

where $G_{0}=e^{2} / h$. In the case of weak tunneling, $G_{L, R} \ll G_{0}$, the co-tunneling mechanism gives only a small correction to the peak value given by Eq. (1). However, it dominates away from peaks at $T \rightarrow 0$.

At low temperatures and at the gate voltage near the peak position, i.e., at $T, \Delta E \ll$ $e^{2} / 2 C$, the tunneling matrix elements are renormalized and grow logarithmically [ $\left.\mathbb{8}\right]$. This can be seen in the following way [9]. On resonance, the state with $n$ electrons in the quantum dot and that with $n+1$ electrons have the same electrostatic energy, i.e., $\Delta E=0$. These two states can be regarded as up and down states of a fictitious impurity "spin" $S=\frac{1}{2}$, and we may discard all other states. If we also ascribe up (down) "spin" to the electrons in the leads (dot), then each tunneling event flips "spins" of both the tunneling electron and the impurity. Thus, the tunneling Hamiltonian can be interpreted as "spin"-flip scattering on an impurity. Hence the tunneling problem is mapped to an anisotropic multi-channel Kondo problem, in which the number of the channels (flavors) is equal to the total number of 1D modes in all the QPCs.

In the leading logarithmic approximation [8,9] one can substitute into Eq. (11) the renormalized $\tilde{G}_{L}$ and $\tilde{G}_{R}$ obtained from the scaling equations for the anisotropic Kondo model,

$$
\tilde{G}_{L(R)}=\frac{G_{L(R)}}{\cos \left[\sqrt{G_{L(R)} / 2 \pi^{2} G_{0}} \ln \left(E_{C} / T\right)\right]} .
$$

If $G_{L}$ and $G_{R}$ are equal, they grow together under the renormalization, and the peak conductance will be of the order of $G_{0}$ at low temperature 10. When $G_{L}$ is initially smaller than $G_{R}$, however, they first increase together according to (3), but then $G_{L}$ starts to decrease to zero whereas $G_{R}$ keeps increasing to $G_{0}$. This is because the fixed point of the multi-channel Kondo problem is unstable against a perturbation breaking the flavor symmetry [11. Thus the total conductance in this case shows a nonmonotonic temperature dependence and goes to zero in the low-temperature limit.

Below we concentrate on the case of strong tunneling, and find much stronger renormalizations of conductance than the ones given by Eq. (3). The system we study is a quantum dot connected to two external leads by single-mode QPCs. We consider the low-temperature case $T \ll E_{C}$, but assume that the level spacing in the dot is much smaller than the temperature. The latter assumption is usually satisfied for reasonably large quantum dots. It means that the phase coherence in transport of electrons from one QPC to the other is destroyed by thermal fluctuations, and one can neglect the corresponding processes of elastic co-tunneling [7]. Since the transport through a single-mode QPC is essentially one dimensional (1D), we may introduce for each QPC an effective 1D model with linearized dispersion relation [12]. We further assume that the Coulomb repulsion can be described by the charging energy $Q^{2} / 2 C$ because of good screening in the quantum dot, and use point-like backward-scattering potential to model reflection at the QPCs. The two 1D systems are coupled by the charging energy. The effective Hamiltonian is

$$
H=v_{F} \int_{-\infty}^{\infty} d x \sum_{j=L, R} \sum_{\sigma=\uparrow, \downarrow}\left[\psi_{j, 1, \sigma}^{\dagger}(x)\left(i \partial_{x}-k_{F}\right) \psi_{j, 1, \sigma}(x)-\psi_{j, 2, \sigma}^{\dagger}(x)\left(i \partial_{x}+k_{F}\right) \psi_{j, 2, \sigma}(x)\right]+\frac{(Q-e N)^{2}}{2 C}
$$




$$
\begin{aligned}
+v_{F} \sum_{\sigma=\uparrow, \downarrow}\left\{\left|r_{L}\right|\left[\psi_{L, 1, \sigma}^{\dagger}(0) \psi_{L, 2, \sigma}(0)+\psi_{L, 2, \sigma}^{\dagger}(0) \psi_{L, 1, \sigma}(0)\right]\right. \\
\left.+\left|r_{R}\right|\left[\psi_{R, 1, \sigma}^{\dagger}(0) \psi_{R, 2, \sigma}(0)+\psi_{R, 2, \sigma}^{\dagger}(0) \psi_{R, 1, \sigma}(0)\right]\right\},
\end{aligned}
$$

where $\psi_{L, 1(2), \sigma}(x)$ is the field operator of a left-going (right-going) electron near the left QPC, $\psi_{R, 1(2), \sigma}(x)$ is that of an electron near the right QPC; dimensionless parameter $N$ is proportional to the gate voltage. The charge in the quantum dot is given by

$$
\begin{aligned}
Q=e \int_{0}^{\infty} \sum_{d=1,2} \sum_{\sigma=\uparrow, \downarrow}[ & : \psi_{L, d, \sigma}^{\dagger}(x) \psi_{L, d, \sigma}(x): \\
& \left.+: \psi_{R, d, \sigma}^{\dagger}(-x) \psi_{R, d, \sigma}(-x):\right] d x .
\end{aligned}
$$

We first consider the case of spinless fermions, which turns out to be equivalent to the two-channel Kondo problem. This case may be realized experimentally by applying a magnetic field parallel to the 2DEG to allow only spin-up electrons to transmit through the QPCs. Following the standard procedure [13], we bosonize the Hamiltonian (4):

$$
\begin{aligned}
H= & \frac{v_{F}}{2} \int_{-\infty}^{\infty} d x \sum_{j=L, R}\left(\frac{1}{\pi}\left[\partial_{x} \phi_{j}(x)\right]^{2}+\pi\left[\Pi_{j}(x)\right]^{2}\right) \\
& +\frac{E_{C}}{\pi^{2}}\left[\phi_{R}(0)-\phi_{L}(0)-\pi N\right]^{2} \\
& +\frac{D\left|r_{L}\right|}{\pi} \cos \left[2 \phi_{L}(0)\right]+\frac{D\left|r_{R}\right|}{\pi} \cos \left[2 \phi_{R}(0)\right],
\end{aligned}
$$

where $\phi_{j}(x)$ is a phase field describing charge density fluctuations, $\left[\phi_{j}(x), \Pi_{k}(y)\right]=i \delta_{j, k} \delta(x-$ $y$ ), and $D$ is the high-energy cutoff (bandwidth). We assume that the reflection amplitudes are small, $\left|r_{L(R)}\right| \ll 1$.

The current through the quantum dot is $I=(e / 2 \pi) \partial_{t}\left[\phi_{L}(0)+\phi_{R}(0)\right]$, and the conductance $G$ is calculated using the Kubo formula. Up to the second order in $r_{L(R)}$ we obtain

$$
\begin{aligned}
G & =\frac{e^{2}}{2 h}\left(1-\frac{\pi \Gamma_{0}(N)}{4 T}\right), \\
\Gamma_{0}(N) & =\frac{2 \gamma E_{C}}{\pi^{2}}\left[\left|r_{L}\right|^{2}+\left|r_{R}\right|^{2}+2\left|r_{L}\right|\left|r_{R}\right| \cos (2 \pi N)\right],
\end{aligned}
$$

where $\gamma=e^{\mathbf{C}}$, with $\mathbf{C}=0.5772 \ldots$ being the Euler's constant. We see that the second term diverges at low temperature [14], unless $\left|r_{L}\right|=\left|r_{R}\right|$, and $N$ is a half-integer [15]. This indicates that the higher-order terms in $|r|$ should be taken into account in a proper way. Since the charging mode $\phi_{R}-\phi_{L}$ is massive due to the charging energy, we may integrate it out to obtain an effective Hamiltonian for the current mode, $\phi_{L}+\phi_{R}$. The resulting Hamiltonian is equivalent to that of a single impurity in the $g=\frac{1}{2}$ Luttinger liquid [16,17], which can be solved exactly [16,18]. We use an alternative exact solution [5] and fermionize the problem to the following quadratic form,

$$
H=\int d k\left[\xi_{k} c_{k}^{\dagger} c_{k}-\left(\lambda c_{k}^{\dagger}\left(c+c^{\dagger}\right)+\lambda^{*}\left(c+c^{\dagger}\right) c_{k}\right)\right]
$$


where $c_{k}$ and $c$ are fermions, $\xi_{k}=v_{F} k$, and parameter $\lambda=\left(\gamma v_{F} E_{C} / 2 \pi^{3}\right)^{1 / 2}\left(\left|r_{L}\right| e^{-i \pi N}+\right.$ $\left.\left|r_{R}\right| e^{i \pi N}\right)$. The current is now given by $I=e v_{F} \int: c_{k_{1}}^{\dagger} c_{k_{2}}: d k_{1} d k_{2}$. After some algebra we get the conductance (Fig. 2)

$$
G=\frac{e^{2}}{2 h}\left[1-\int_{-\infty}^{\infty} d E\left(-\frac{d f}{d E}\right) \frac{\Gamma_{0}^{2}(N)}{E^{2}+\Gamma_{0}^{2}(N)}\right],
$$

where $f(E)=\left(e^{E / T}+1\right)^{-1}$. We see that even for half-integer $N$, i.e., on resonance, the conductance vanishes as $T^{2}$ if $\left|r_{L}\right|$ and $\left|r_{R}\right|$ are not equal. In the off-resonance case the conductance also vanishes as $T^{2}$, in agreement with the result (2) of the inelastic co-tunneling theory. On resonance, at $\left|r_{L}\right|=\left|r_{R}\right|$, the conductance equals $e^{2} / 2 h$. As expected, the conductance (9) coincides with the one for a single impurity in the $g=\frac{1}{2}$ Luttinger liquid 16.

We next take into account the spins of electrons. The bosonized form of the Hamiltonian (四) is

$$
\begin{aligned}
H= & \frac{v_{F}}{2} \int d x \sum_{j=L, R}\left(\frac{1}{\pi}\left[\partial_{x} \phi_{j, c}(x)\right]^{2}+\pi\left[\Pi_{j, c}(x)\right]^{2}+\frac{1}{\pi}\left[\partial_{x} \phi_{j, s}(x)\right]^{2}+\pi\left[\Pi_{j, s}(x)\right]^{2}\right) \\
& +\frac{2 E_{C}}{\pi^{2}}\left(\phi_{R, c}(0)-\phi_{L, c}(0)-\frac{\pi}{\sqrt{2}} N\right)^{2} \\
& +\frac{2 D\left|r_{L}\right|}{\pi} \cos \left(\sqrt{2} \phi_{L, c}(0)\right) \cos \left(\sqrt{2} \phi_{L, s}(0)\right)+\frac{2 D\left|r_{R}\right|}{\pi} \cos \left(\sqrt{2} \phi_{R, c}(0)\right) \cos \left(\sqrt{2} \phi_{R, s}(0)\right),
\end{aligned}
$$

where $\phi_{j, c}(x)$ and $\phi_{j, s}(x)$ are the phase fields for the charge and spin density fluctuations. The electric current is given by $I=(e / \sqrt{2} \pi) \partial_{t}\left[\phi_{L, c}(0)+\phi_{R, c}(0)\right]$. Up to the order $|r|^{2}$ the conductance is

$$
G=\frac{e^{2}}{h}\left(1-\frac{2 \Gamma\left(\frac{3}{4}\right)}{\Gamma\left(\frac{1}{4}\right)} \sqrt{\frac{\gamma E_{C}}{\pi T}}\left(\left|r_{L}\right|^{2}+\left|r_{R}\right|^{2}\right)\right) .
$$

The term proportional to $|r|^{2}$ has no dependence on $N$ and diverges as $1 / \sqrt{T}$ at $T \rightarrow 0$, indicating that $\left|r_{L}\right|=\left|r_{R}\right|=0$ is an unstable fixed point even on resonance, in contrast to the spinless case discussed above. If $\left|r_{L}\right|=\left|r_{R}\right|>0$, and $N$ is a half-integer, then the system will be renormalized toward the fixed point of the four-channel Kondo problem. In fact, after a series of transformations we could map the Hamiltonian to the one which appeared in the study of the four-channel Kondo problem [19]. Unfortunately, in this case we cannot sum up analytically all the higher order divergent terms.

On the other hand, we can still find the low-temperature asymptotics of the conductance in a realistic case when the reflection amplitudes $r_{L}$ and $r_{R}$ are not precisely equal. In this case, as we already mentioned above, one can use mapping to the multi-channel Kondo model to identify the stable fixed point of the problem. Since the channel anisotropy is a relevant perturbation, in the low-energy limit the larger of the two reflection amplitudes, say $\left|r_{L}\right|$, is renormalized to unity (weak-tunneling limit), whereas $r_{R}$ is renormalized to zero. Thus it is meaningful to study the case where the transmission amplitude $t_{L}$ of the left QPC and the reflection amplitude $r_{R}$ of the right QPC are very small. This limit can also be easily realized experimentally by tuning voltages on the gates controlling the QPCs, Fig. 1. 
We will calculate the conductance in the lowest order in the tunneling probability through the left barrier. The problem is therefore to calculate the renormalization of the tunneling density of states for the left-lead electrons. The renormalization is due to the electrostatic coupling between the two 1D electron systems describing the two QPCs, see Eq. (4). When an electron tunnels through the left barrier, the number of particles in the dot changes by 1 , which means that the system of electrons of the right QPC is no longer in the ground state. This leads to the suppression of tunneling due to the orthogonality catastrophe. The resulting tunneling conductance is [14]

$$
G=G_{L} \int_{-\infty}^{\infty} d \omega \frac{\frac{\omega}{T} e^{\omega / T}-e^{\omega / T}+1}{\left(e^{\omega / T}-1\right)^{2}} \operatorname{Re} K(\omega),
$$

where $G_{L} \ll G_{0}$ is the conductance of the left barrier, and $K$ is the correlator of the operators shifting the potential for electrons in the right QPC: $N \rightarrow N+1$.

At $r_{R}=0$ the Hamiltonian for the right QPC is quadratic, and the correlator $K(\omega)$ can be found exactly. In this case only the charge mode $\phi_{R, c}$ is affected by the shift operator, and $K(\omega)=K_{c}(\omega)=\left(\pi^{2} / \gamma E_{C}\right) f(-\omega)$. The resulting conductance is

$$
G=\frac{\pi^{3} T}{8 \gamma E_{C}} G_{L}, \quad r_{R}=0 .
$$

An attempt to treat the reflection amplitude $r_{R}$ perturbatively leads to the correction, similar to the second term in Eq. (77), which diverges at $T \rightarrow 0$, [14]. To get the nonperturbative result, one can first notice that the system of electrons in the right QPC has only two channels. Thus one can use the same technique [5] as in the derivation of Eq. (9). First, we integrate out the charge modes, and then the remaining Hamiltonian is fermionized to the form (81). Clearly, the tunneling of an electron through the left barrier affects the fermionized spin modes by shifting $N \rightarrow N+1$ and thus changing the sign of $\lambda$ in Eq. (8). For the quadratic Hamiltonian (8) the calculation of the corresponding contribution $K_{s}(\omega)=4 f(-\omega) \Gamma_{R} /\left(\Gamma_{R}^{2}+\omega^{2}\right)$ to the correlator $K(t)=K_{c}(t) K_{s}(t)$ is straightforward; here $\Gamma_{R}=\left(8 \gamma / \pi^{2}\right) E_{C}\left|r_{R}\right|^{2} \cos ^{2}(\pi N)$. As a result, we get

$$
G=\frac{\pi^{2} T G_{L}}{8 \gamma E_{C}} \int_{-\infty}^{\infty} d E \frac{\Gamma_{R}}{E^{2}+\Gamma_{R}^{2}} \frac{1+(E / \pi T)^{2}}{\cosh ^{2}(E / 2 T)} .
$$

When $N$ is not a half-integer, i.e., off resonance, the conductance vanishes as $T^{2}$, which is again in agreement with the theory of inelastic co-tunneling. On the other hand, on resonance the conductance has a linear temperature dependence (13).

In summary, we have developed a theory for the inelastic transport through a quantum dot via single-mode quantum point contacts in the strong-tunneling regime. We have obtained analytic expressions for the peaks in linear conductance. At any tunneling strength, between the peaks the conductance vanishes at low temperatures as $T^{2}$, in agreement with the theory of inelastic co-tunneling developed for the weak-tunneling case [7]. In the case of symmetric barriers the resonant value of conductance is $\sim e^{2} / h$.

We are grateful to L. I. Glazman and P. A. Lee for helpful discussions. The work was sponsored by Joint Services Electronics Program Contract DAAL03-92-C-0001. 


\section{REFERENCES}

* On leave of absence from Department of Applied Physics, University of Tokyo, Hongo, Tokyo 113, Japan.

[1] For a review, see D. V. Averin and K. K. Likharev, in Mesoscopic Phenomena in Solids, edited by B. Altshuler, P. A. Lee, and R. A. Webb (Elsevier, Amsterdam, 1991).

[2] M. A. Kastner, Rev. Mod. Phys. 64, 849 (1992).

[3] N. C. van der Vaart et al., Physica B 189, 99 (1993).

[4] C. Pasquier et al., Phys. Rev. Lett. 70, 69 (1993).

[5] K. A. Matveev, Phys. Rev. B 51, 1743 (1995).

[6] L. I. Glazman and R. I. Shekhter, J. Phys. Conden. Matter 1, 5811 (1989).

[7] D. V. Averin and Yu. V. Nazarov, Phys. Rev. Lett. 65, 2446 (1990); D. V. Averin and A. A. Odintsov, Phys. Lett. A 140, 251 (1989).

[8] L. I. Glazman and K. A. Matveev, Zh. Eksp. Teor. Fiz. 98, 1834 (1990) [Sov. Phys. JETP 71, 1031 (1990)].

[9] K. A. Matveev, Zh. Eksp. Teor. Fiz. 99, 1598 (1991) [Sov. Phys. JETP 72, 892 (1991)].

[10] The leading logarithm approximation (3) is valid only as long as $\tilde{G}_{L, R} \ll G_{0}$.

[11] P. Nozières and A. Blandin, J. Phys. (Paris) 41, 193 (1980); A. I. Larkin and V. I. Melnikov, Zh. Eksp. Teor. Fiz. 61, 1231 (1971) [Sov. Phys. JETP 34, 656 (1972)].

[12] The detailed discussion on this reduction to effective one-dimensional models can be found in Ref. [5]. A similar model has been used earlier by K. Flensberg [14].

[13] J. Sólyom, Adv. Phys. 28, 201 (1979); V. J. Emery, in Highly Conducting OneDimensional Solids, eds. J. Devreese et al. (Plenum, New York, 1979).

[14] K. Flensberg, Phys. Rev. B 48, 11156 (1993).

[15] A. Furusaki and N. Nagaosa, Phys. Rev. B 47, 3827 (1993).

[16] C. L. Kane and M. P. A. Fisher, Phys. Rev. B 46, 15233 (1992).

[17] Since half of the degrees of freedom, the charging mode, is frozen due to the charging effect at $T<E_{C}$, the parameter $g$ becomes half of the original value $g=1$ [15].

[18] F. Guinea, Phys. Rev. B 32, 7518 (1985).

[19] M. Fabrizio and A. O. Gogolin, Phys. Rev. B 50, 17732 (1994). 


\section{FIGURES}

FIG. 1. Schematic view of a quantum dot connected to two bulk $2 \mathrm{D}$ electrodes. The dot is formed by applying negative voltage to the gates (shaded). Solid line shows the boundary of the 2D electron gas (2DEG). Electrostatic conditions in the dot are controlled by the voltage applied to the central gates. Voltage $V_{l, r}$ applied to the auxiliary gates controls the transmission probability through the left and right constrictions.

FIG. 2. Conductance (9) as a function of the dimensionless gate voltage $N$ for the symmetric case, $\left|r_{L}\right|=\left|r_{R}\right|=0.3$. The three curves are calculated for $E_{C} / T=1,10$, and 100 . 


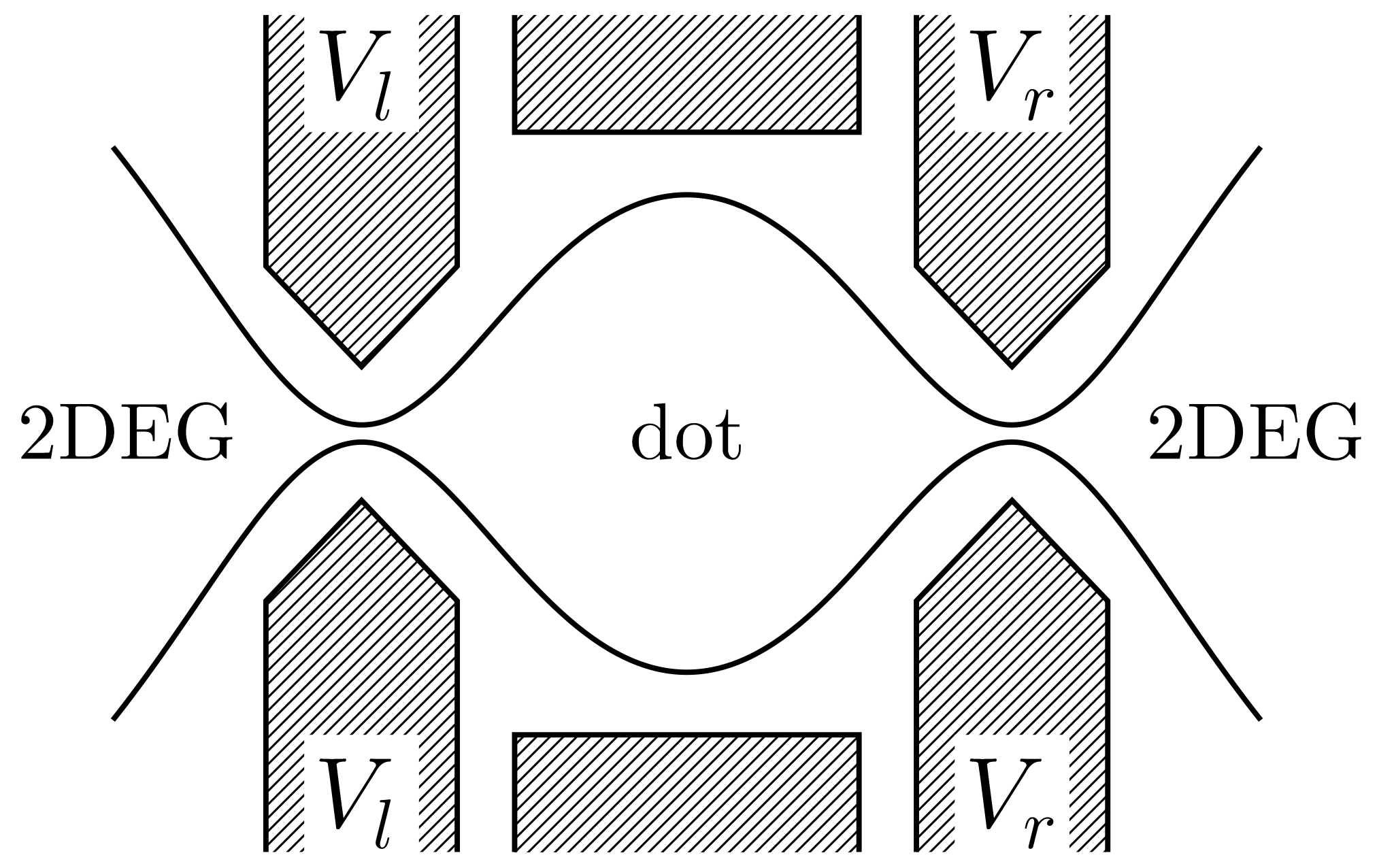




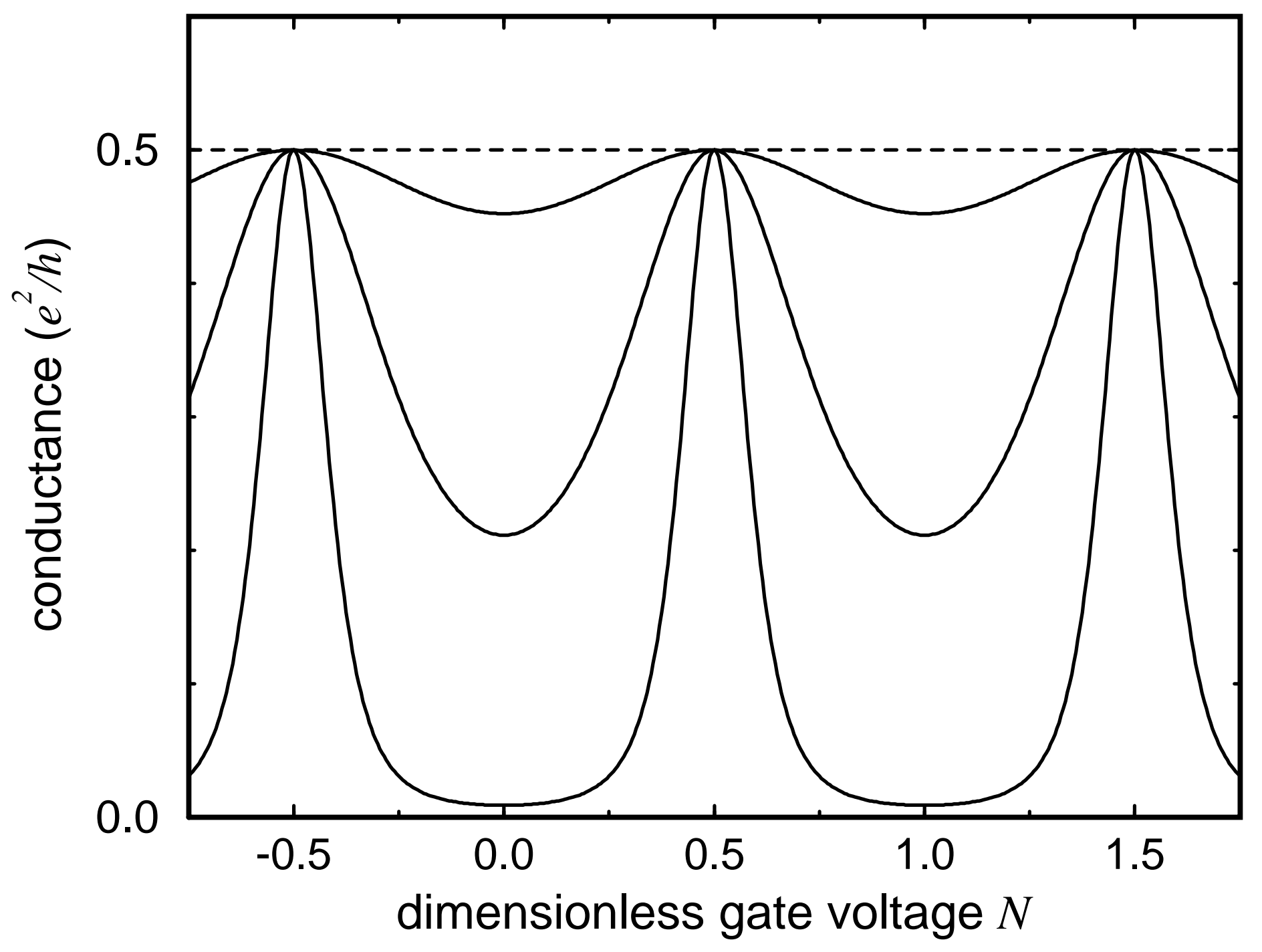

